\title{
The Quantum Compass Model on the Square Lattice
}

\author{
Julien Dorier, ${ }^{1}$ Federico Becca ${ }^{2}$ and Frédéric Mila ${ }^{1}$ \\ 1 Institut de Théorie des Phénomènes Physiques, \\ Ecole Polytechnique Fédérale de Lausanne (EPFL), CH-1015 Lausanne, Switzerland \\ 2 INFM-Democritos, National Simulation Centre, and SISSA I-34014 Trieste, Italy
}

(Dated: October 23, 2018)

\begin{abstract}
Using exact diagonalizations, Green's function Monte Carlo simulations and high-order perturbation theory, we study the low-energy properties of the two-dimensional spin- $1 / 2$ compass model on the square lattice defined by the Hamiltonian $H=-\sum_{\boldsymbol{r}}\left(J_{x} \sigma_{\boldsymbol{r}}^{x} \sigma_{\boldsymbol{r}+\boldsymbol{e}_{x}}+J_{z} \sigma_{\boldsymbol{r}}^{z} \sigma_{\boldsymbol{r}+\boldsymbol{e}_{z}}^{z}\right)$. When $J_{x} \neq J_{z}$, we show that, on clusters of dimension $L \times L$, the low-energy spectrum consists of $2^{L}$ states which collapse onto each other exponentially fast with $L$, a conclusion that remains true arbitrarily close to $J_{x}=J_{z}$. At that point, we show that an even larger number of states collapse exponentially fast with $L$ onto the ground state, and we present numerical evidence that this number is precisely $2 \times 2^{L}$. We also extend the symmetry analysis of the model to arbitrary spins and show that the two-fold degeneracy of all eigenstates remains true for arbitrary half-integer spins but does not apply to integer spins, in which cases eigenstates are generically non degenerate, a result confirmed by exact diagonalizations in the spin-1 case. Implications for Mott insulators and Josephson junction arrays are briefly discussed.
\end{abstract}

PACS numbers: 71.27.+a,75.30.Ds,03.67.Lx

\section{INTRODUCTION}

Building on the deep understanding of the Heisenberg and other models of magnetism, it is a very common practice to describe discrete degrees of freedom as pseudospins, with the hope to gain insight from the form of the resulting magnetic model. A well-known example of considerable current interest shows up in the context of Mott insulators with orbital degeneracy: In an octahedral environment, the degeneracy of the $d$ electrons is only partially lifted, and the remaining orbital degree of freedom is often described as a spin-1/2 or 1 for $e_{g}$ and $t_{2 g}$ electrons, respectively [1, 2]. However, the situation is in general less simple that one might hope. Indeed, as already emphasized by Kugel and Khomskii, the symmetry of pseudo-spin Hamiltonians is in general much lower than $\mathrm{SU}(2)$, and there are cases where the properties of the resulting model are poorly understood. This is in particular the case of models where the anisotropy of the coupling in spin space is related to the orientation of the bond in real space. The simplest version of such a model on the square lattice is defined by the Hamiltonian

$$
H=-J \sum_{\boldsymbol{r}}\left(\tau_{\boldsymbol{r}}^{x} \tau_{\boldsymbol{r}+\boldsymbol{e}_{x}}^{x}+\tau_{\boldsymbol{r}}^{z} \tau_{\boldsymbol{r}+\boldsymbol{e}_{z}}^{z}\right),
$$

where $\tau_{\boldsymbol{r}}^{\alpha}$ are the $x$ and $z$ components of a pseudo-spin operator. By analogy with the dipolar coupling between compass needles, this model has been called the compass model by Kugel and Khomskii [2]. Realistic models of orbital degeneracy are usually more complicated in several respects. In particular, the spins and pseudo-spins are usually coupled. Pure orbital models can be of direct relevance though if the spins order ferromagnetically, as recently argued by Mostovoy and Khomskii in the context of $\mathrm{NaNiO}_{2}[3]$. The precise symmetry is also usually more complicated than the simple case of this Hamilto- nian, but we will nevertheless concentrate on that model, considering it as a minimal model rather than a realistic one.

Interestingly, such models have appeared in other contexts as well. First, even in Mott insulators without orbital degeneracy, extra degrees of freedom can appear if the system is frustrated and, for instance, consists of spin- $1 / 2$ coupled triangles, like in the trimerized kagome lattice, in which case the chirality that keeps track of the extra degeneracy of each triangle plays a role similar to that of orbitals 4]. In a magnetic field, this model has been predicted to exhibit a magnetization plateau at $1 / 3[\underline{5}$ ], and the low-energy properties inside the plateau can be described by a kind of compass model $[\underline{6}]$.

More recently, the model of Eq. (10) with anisotropic couplings along the $x$ and $z$ directions has been proposed by Douçot and collaborators in the context of Josephson junction arrays [7].

Despite its deceptive simplicity, the model of Eq. (11) is a formidable challenge, in many respect comparable to very frustrated magnets. To see this, let us follow Ref. 8] and consider the classical version of the model, in which spins are considered as classical vectors. In that case, as shown by Nussinov et al., the ground state is highly degenerate, as in very frustrated magnets. First of all, all ferromagnetic states are degenerate, regardless of the relative orientation of the spins with respect to the lattice, as can be easily checked from Eq. (1). In addition, from any ferromagnetic state, one can construct other states by flipping all spins of a $z$ column with respect to an $x$ mirror, or equivalently by flipping all spins of an $x$ line with respect to a $z$ mirror. Since all these operations can be performed simultaneously and in any order, they generate a discrete degeneracy of order $2^{L}$.

The effects of thermal fluctuations on the classical model have been convincingly identified by analytical and 
numerical approaches. Nussinov et al. have shown that an order by disorder mechanism is expected to lift the rotational degeneracy and to select states in which the spins point along the $x$ or $z$ axis, leading to a nematic ground state since lines or columns of spins are still free to flip. Using extensive Monte Carlo simulations, Mishra et al. have shown that the two possible orientations along $x$ or $z$ lead to an effective Ising order parameter, and that the model undergoes a finite temperature phase transition of the Ising type 9].

On the other hand, the understanding of the spin- $1 / 2$ version of the model is still preliminary. Most of the results have been obtained by Douçot et al. 7] in their analysis of a generalized version of the model of Eq. (1) defined by the Hamiltonian

$$
H=-\sum_{\boldsymbol{r}}\left(J_{x} \tau_{\boldsymbol{r}}^{x} \tau_{\boldsymbol{r}+\boldsymbol{e}_{x}}^{x}+J_{z} \tau_{\boldsymbol{r}}^{z} \tau_{\boldsymbol{r}+\boldsymbol{e}_{z}}^{z}\right),
$$

in which the couplings along the $x$ and $z$ directions can take different values. Using elegant symmetry arguments, Douçot et al. have shown that all eigenstates must be at least two-fold degenerate. They have also shown that in the strongly asymmetric case $\left(J_{x} / J_{z} \ll 1\right.$ or $\left.J_{z} / J_{x} \ll 1\right)$, the $2^{L}$ states that evolve adiabatically from the $2^{L}$ ground states of the decoupled Ising chain case $\left(J_{x}=0\right.$ or $\left.J_{z}=0\right)$ should collapse onto each other. This perturbative argument does not apply close to $J_{x}=J_{z}$ though, and whether this remains true in the isotropic case could not be decided. Note that this is an important issue in the context of quantum bits in which they came accross this model since the presence of a gap would help to protect the q-bits. More recently, Nussinov and Fradkin 10] have shown that these models are dual to models of $p+i p$ superconducting arrays, and have discussed general properties of order parameters and phase transitions.

In this paper, we concentrate on the zero-temperature properties of the quantum version of the model. We start by a semi-classical analysis of the model of Eq. (1) and show that, as in many frustrated magnets, quantum fluctuations essentially have the same effect as thermal fluctuations regarding the lifting of classical degeneracy (section [II). We then turn to an extensive analysis of the model of Eq. (2) in the spin- $1 / 2$ case. As we shall see, there is no minus sign problem in the Green's function implementation of quantum Monte Carlo, which allows one to study the ground state properties of very large clusters, i.e., up to $17 \times 17$. Combined with the large number of symmetries, hence of different symmetry sectors that can be studied independently, this allowed us to reach definite conclusions regarding the low energy spectrum, conclusions that agree with high order perturbation theory. These results are presented in section III Finally, the symmetry analysis of Douçot et al. is extended to larger spins in section IV with the conclusion that integer and half-integer spins behave once more quite differently. Some implications of the present results are discussed in the last section of the paper.

\section{SEMI-CLASSICAL COMPASS MODEL}

In order to have a first insight into the properties of the quantum version of the compass model, we have performed a spin-wave analysis in the symmetric case defined by Eq. (1). In that respect, it is useful to emphasize that, as noticed in Refs. [8, 9], the degeneracy is partly accidental and partly due to symmetry. Indeed, in addition to the lattice translational symmetries, this model has two types of discrete symmetries: (i) The $Q_{i}$ transformation which flips the $z$ component of all the spins of the column $r_{x}=i$, and the $P_{j}$ transformations which flip the $x$ component of all spins of the line $r_{z}=j$. Note that the transformations $Q_{i}$ (resp. $P_{j}$ ) could be seen as rotations of all the spins of the column (resp. line) about the $\boldsymbol{e}_{x}$ axis (resp. $\boldsymbol{e}_{z}$ ) by an angle $\pi$. (ii) The simultaneous rotation $R_{y}\left(\frac{\pi}{2}\right)$ of all spins and of the lattice about an $\boldsymbol{e}_{y}$ axis by an angle $\frac{\pi}{2}$. Starting from any state, these symmetries generate new states with exactly the same classical energy and the degeneracy associated to these symmetries cannot be lifted by thermal fluctuations [9]. However, the ground state also has an accidental degeneracy: All ferromagnetic states are degenerate regardless of the angle between the spins and the lattice. This degeneracy is not related to a symmetry since the model is not rotationally invariant. Accordingly, it has been found that the thermal fluctuations partially lift this degeneracy via an order-by-disorder mechanism, favouring the $2 \times 2^{L}$ ground states with all spins parallel to $\boldsymbol{e}_{x}$ or $\boldsymbol{e}_{z}$ and implying a directional ordering of the spins. These favoured states are the uniform state with all spins in the direction $\boldsymbol{e}_{x}$ and all the states obtained by applying the symmetries $R_{y}\left(\frac{\pi}{2}\right), P_{j}$ and $Q_{i}$. So, as anticipated, only the accidental degeneracy is lifted by thermal fluctuations.

The same idea applies to quantum fluctuations. Starting from an arbitrary ground state, one can bring it back into a ferromagnetic ground state applying only symmetry operations. But applying symmetry operations does not change the form of the Hamiltonian. The fluctuations around both states will thus have exactly the same form. Then it is sufficient to do the spin-wave expansion around the uniform classical ground states $\boldsymbol{S}_{\boldsymbol{r}}=S \cos \theta_{0} \boldsymbol{e}_{x}+S \sin \theta_{0} \boldsymbol{e}_{z}$. To linear order in $1 / S$, the energy can be brought into the form

$$
H=E_{0}+\frac{1}{2} \sum_{q} \omega_{q}\left(\theta_{0}\right)
$$

with $\omega_{q}\left(\theta_{0}\right)=4 J S \sqrt{1-\cos ^{2} \theta_{0} \cos k_{x}-\sin ^{2} \theta_{0} \cos k_{z}}$, and the resulting ground-state energy is plotted in Fig. 1] As in the classical case with thermal fluctuations, the figure clearly shows that the angles $\theta_{0}=0, \frac{\pi}{2}, \pi, \frac{3 \pi}{2}$ are selected by quantum fluctuations since they minimize the energy. Applying the symmetries $Q_{i}, P_{j}$ and $R_{y}\left(\frac{\pi}{2}\right)$ to these states gives $2 \times 2^{L}$ equivalent favoured states, corresponding to $2^{L}$ states parallel to $\boldsymbol{e}_{x}$ and $2^{L}$ states parallel to $\boldsymbol{e}_{z}$. So, as in the classical case, there is a directional 


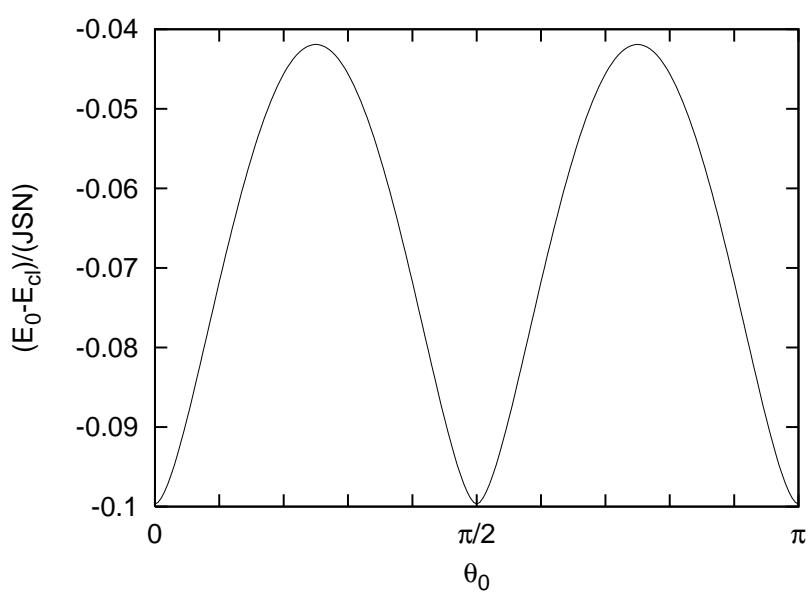

FIG. 1: Ground-state energy of the ferromagnetic states defined by $\boldsymbol{S}_{\boldsymbol{r}}=\boldsymbol{S}\left(\theta_{0}\right)=S \cos \theta_{0} \boldsymbol{e}_{x}+S \sin \theta_{0} \boldsymbol{e}_{z}$ including zeropoint energy as a function of the angle $\theta_{0} . E_{c l}=-J N S^{2}$ is the classical ground-state energy.

ordering of the ground state.

As is many frustrated magnets like the $J_{1}-J_{2}$ model on the square lattice for $J_{2} / J_{1}=1 / 2$, this calculation is not fully consistent since the correction to the magnetization diverges [11]. In the present case, the divergence comes from a line of zero energy along the $k_{x}=0$ direction when $\theta_{0}=0, \pi$ and along the $k_{z}=0$ direction when $\theta_{0}=\frac{\pi}{2}, \frac{3 \pi}{2}$. We have pushed the expansion to next order in $1 / \mathrm{S}$ and checked that a self-consistent mean-field decoupling of the 4-boson terms suppresses the divergence. At this approximation, the spectrum becomes gapped, which does not violate any general theorem since the model does not possess rotational symmetry, and long-range order is preserved even for spin- $1 / 2$.

In the anisotropic case $\left(J_{x} \neq J_{z}\right)$, discrete rotational symmetry of the Hamiltonian as well as continuous rotational symmetry of the ground state are lost, leading to only the $2^{L}$ ground states with rows of parallel spins along the $x$ axis for $J_{x}>J_{z}$ and columns of parallel spins along the $z$ axis for $J_{x}<J_{z}$. Since these $2^{L}$ classical ground states are related by symmetries of the Hamiltonian, quantum fluctuations cannot lift this degeneracy.

\section{QUANTUM COMPASS MODEL: SPIN-1/2}

In this section, we turn to the spin- $1 / 2$ case for which we write the Hamiltonian

$$
H=-\sum_{\boldsymbol{r}}\left(J_{x} \sigma_{\boldsymbol{r}}^{x} \sigma_{\boldsymbol{r}+\boldsymbol{e}_{x}}^{x}+J_{z} \sigma_{\boldsymbol{r}}^{z} \sigma_{\boldsymbol{r}+\boldsymbol{e}_{z}}^{z}\right),
$$

where $\sigma_{\boldsymbol{r}}^{x}$ and $\sigma_{\boldsymbol{r}}^{z}$ are Pauli matrices acting on the spin at site $\boldsymbol{r}$. In the following, we will use the parametrization of the exchange integrals $J_{x}=J \cos \theta$ and $J_{z}=J \sin \theta$ with $\theta \in\left[0, \frac{\pi}{2}\right]$, and we will study the model on $N$-site square clusters of dimension $L \times L$.
Taking all symmetries into account, exact diagonalizations could be performed up to $L=5$. As we shall see, this is not sufficient to draw conclusions regarding the degeneracy of the ground state in the thermodynamic limit close to $J_{x}=J_{z}$. However, this model has the very interesting property that all non-diagonal matrix elements are negative. This has allowed us to implement the Green's function Monte Carlo algorithm 12], which gives access to the ground-state energy in a given symmetry sector, and to reach clusters up to $L=17$. Besides, one can choose quantum numbers so that all relevant low-energy states are ground states of a given symmetry sector. To see how this works, let us look more closely at the symmetries of the model.

In addition to the lattice translation symmetries, the Hamiltonian (4) has another type of discrete symmetries. The first one corresponds to the operators $Q_{i}=\prod_{j} \sigma_{i, j}^{x}$, which are the products of the $\sigma_{r}^{x}$ on one column $\left(r_{x}=i\right)$. These operations correspond to a rotation by an angle $\pi$ about the $\boldsymbol{e}_{x}$ axis of all the spins of a given column: $Q_{i}^{-1} \sigma_{i, j}^{y, z} Q_{i}=-\sigma_{i, j}^{y, z}$ and $Q_{i}^{-1} \sigma_{i, j}^{x} Q_{i}=\sigma_{i, j}^{x}$. The second one corresponds to the operators $P_{j}=\prod_{i} \sigma_{i, j}^{z}$, which are the products of the $\sigma_{\boldsymbol{r}}^{z}$ on one line $\left(r_{z}=j\right)$, and which correspond to a rotation by an angle $\pi$ about the $\boldsymbol{e}_{z}$ axis of all the spins of a given line: $P_{j}^{-1} \sigma_{i, j}^{x, y} P_{j}=-\sigma_{i, j}^{x, y}$ and $P_{j}^{-1} \sigma_{i, j}^{z} P_{j}=\sigma_{i, j}^{z}$. In the isotropic limit $J_{x}=J_{z}$, there is one more discrete symmetry: the global rotation $R_{y}\left(\frac{\pi}{2}\right)$ of all spins and lattice about the $\boldsymbol{e}_{y}$ axis by an angle $\frac{\pi}{2}$.

As emphasized by Douçot et al., the $P_{j}$ 's commute with each other, as well as the $Q_{i}{ }^{\prime}$ s, but $\left[Q_{i}, P_{j}\right] \neq 0$ $\forall i, j$. This has two remarkable consequences: First of all, all eigenstates must be two-fold degenerate. Besides, we can choose either the $P_{j}$ 's or the $Q_{i}$ 's to define symmetry sectors in which the Hamiltonian can be independently diagonalized. Since $P_{j}^{2}=1$ and $P_{j}^{\dagger}=P_{j}$, the eigenvalues of $P_{j}$ are $p_{j}= \pm 1$, the same being true for the $Q_{i}$ 's. Thus the Hamiltonian can be diagonalized in the symmetry sectors characterized by the set $\left(p_{1}, \cdots, p_{L}\right), p_{i}= \pm 1$, or alternatively in the sectors defined by the eigenvalues of $Q_{i}$ and characterized by the set $\left(q_{1}, \cdots, q_{L}\right), q_{i}= \pm 1$.

To see how this works, let us start from the trivial case $J_{x}=0$. The model then consists of a set of decoupled Ising columns with eigenstates $|\boldsymbol{m}\rangle=\left|m_{1,1}\right\rangle \otimes\left|m_{1,2}\right\rangle \otimes$ $\cdots \otimes\left|m_{L, L}\right\rangle$, where $m_{i, j}= \pm 1$ is the eigenvalue of $\sigma_{i, j}^{z}$. The ground state manifold contains $2^{L}$ states defined by $m_{i, 1}=\cdots=m_{i, L}= \pm 1, i=1, \ldots, L$. Clearly, all $|\boldsymbol{m}\rangle$ states are eigenstates of the $P_{j}$ 's. Now, in any of the ground state, all $p_{j}$ 's are equal since all lines are identical, and the ground state manifold consists of $2^{L-1}$ states in the sector $p_{1}=\cdots=p_{L}=+1$ and $2^{L-1}$ states in the sector $p_{1}=\cdots=p_{L}=-1$.

The classification according to the $Q_{i}$ 's is quite different. First, note that the states $\{|\boldsymbol{m}\rangle\}$ are not eigenstates of $Q_{i}$. However, if we denote by $|\uparrow\rangle_{i}$ (resp. $|\downarrow\rangle_{i}$ ) the ground state of column $i$ with all the spins up (resp. down), then one can define two new ground states by $|+\rangle_{i}=\frac{1}{\sqrt{2}}\left(|\uparrow\rangle_{i}+|\downarrow\rangle_{i}\right)$ and $|-\rangle_{i}=\frac{1}{\sqrt{2}}\left(|\uparrow\rangle_{i}-|\downarrow\rangle_{i}\right)$. 
These new states are eignestates of $Q_{i}$ with eigenvalues \pm 1 respectively. So $q_{i}$ can be either -1 or +1 for each column independently, and the ground state manifold has one member in each of the $2^{L}$ sectors $\left(q_{1}, \cdots, q_{L}\right)$, $q_{i}= \pm 1$.

The case $J_{z}=0$ is connected to the case $J_{x}=0$ by the rotation symmetry $R_{y}\left(\frac{\pi}{2}\right)$. Since $R_{y}\left(\frac{\pi}{2}\right)^{-1} P_{j} R_{y}\left(\frac{\pi}{2}\right)=$ $(-1)^{L} Q_{L-j}$ and $R_{y}\left(\frac{\pi}{2}\right)^{-1} Q_{i} R_{y}\left(\frac{\pi}{2}\right)=P_{i}$, the symmetry sectors are interchanged. So the ground state is made up of $2^{L}$ states in each $2^{L}$ sectors $\left(p_{1}, \cdots, p_{L}\right)=$ $( \pm 1, \cdots, \pm 1)$.

So, as announced earlier, to determine the structure of the low-energy spectrum when going away from $J_{x}=0$ or $J_{z}=0$, it is always possible to choose the quantum numbers so that each state is the ground state of a given symmetry sector.

\section{A. Exact diagonalization}

The spectrum of the Hamiltonian (4) versus the asymmetry parameter $\theta$ has been determined for $L=2,3,4$ and 5 using exact diagonalization. Fig. 2 presents the low energy levels for $L=4$ and $L=5$. As expected, when $J_{x}, J_{z} \neq 0$, the ground state is two-fold degenerate with one state in the sector $p_{1}=\cdots=p_{L}=+1$ and the other in the sector $p_{1}=\cdots=p_{L}=-1$. For $0<J_{z}<J_{x}$, the $2^{L}$-fold degeneracy of the ground state at $\theta=0$ (i.e., $\left.J_{z}=0\right)$ is lifted by the $J_{z}$ term of the Hamiltonian. The lowest of these states is in the sector $p_{1}=\cdots=p_{L}= \pm 1$ and the highest in the sector $p_{i}= \pm(-1)^{i}$. The gap between these two states is denoted by $\Delta(\theta)$. As we will see in the following, $\Delta(\theta)$ goes to zero for all $\theta$ in the thermodynamic limit.

In the symmetric case $\left(\theta=\frac{\pi}{4}\right.$, i.e., $\left.J_{x}=J_{z}\right)$, the adiabatic continuation of the degenerate ground states of the $J_{x}=0$ and $J_{z}=0$ cases generates only $2 \times 2^{L}-2$ states, and not $2 \times 2^{L}$ as one might naively expect from the semi-classical case. The reason is that the lowest pair of state is common to the two families of states coming from $J_{x}=0$ and $J_{z}=0$, while all other states cross at $J_{x}=J_{z}$ (see Fig. 22). This does not mean however that the low energy sector has only $2 \times 2^{L}-2$ states for very large systems. In fact, another pair of states is decreasing quite fast toward the ground state as a function of the size. Since these states correspond to the first two-fold degenerate excited state of the sectors $p_{1}=\cdots=p_{L}=1$ and $p_{1}=\cdots=p_{L}=-1$, one can keep track of their energy for all sizes. The gap between the ground state and these states, denoted by $\Delta_{2}$, is plotted in Fig. 3 as a function of $1 / N$. These results are indeed consistent with a vanishing of this gap in the thermodynamic limit [13].

\section{B. Perturbation theory}

Before discussing the results obtained for large clusters with Green's function quantum Monte Carlo, let us see
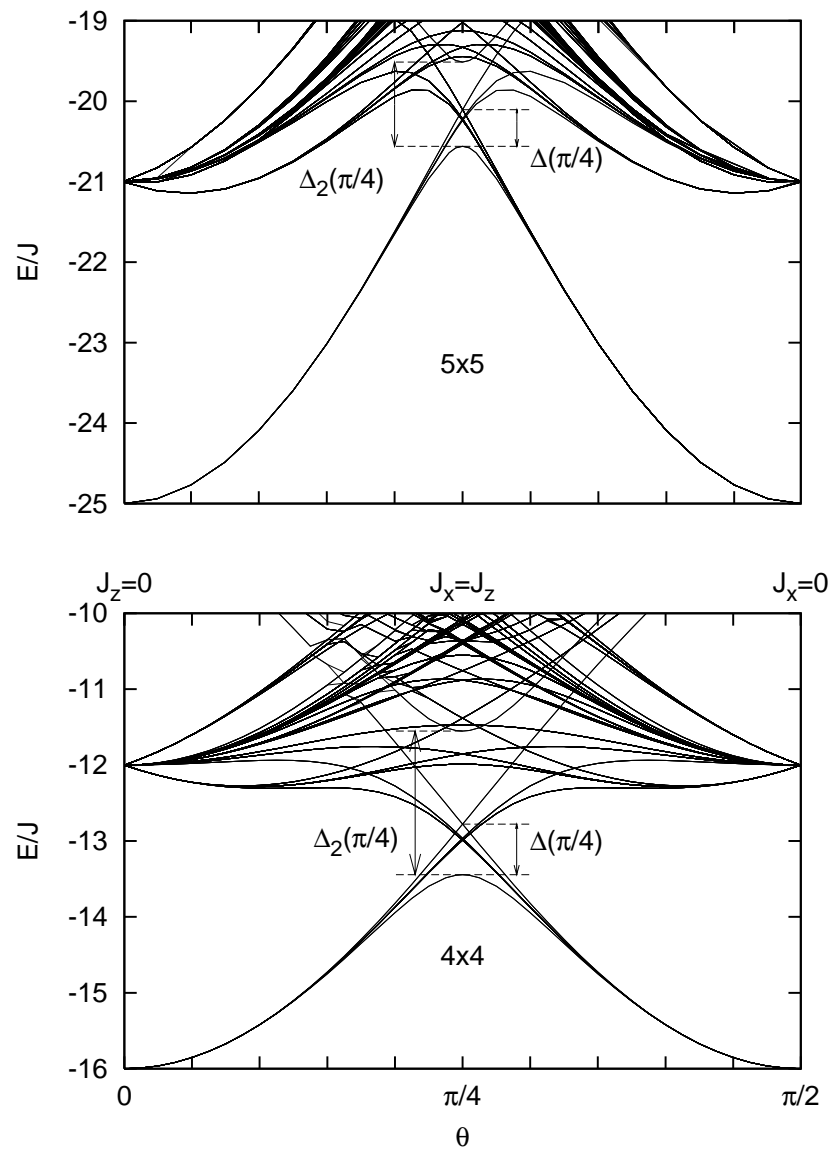

FIG. 2: Energy of the low-lying states versus anisotropy parameter $\theta$ for $4 \times 4$ and $5 \times 5$ lattices obtained by exact diagonalization $\left(J_{x}=J \cos \theta, J_{z}=J \sin \theta\right)$.

what perturbation theory predicts regarding the scaling of the gap $\Delta$ in the limit $J_{z} \ll J_{x}$. To this purpose, the Hamiltonian is written as

$$
H=H_{0}+V,
$$

where $H_{0}=-J_{x} \sum_{\boldsymbol{r}} \sigma_{\boldsymbol{r}}^{x} \sigma_{\boldsymbol{r}+\boldsymbol{e}_{x}}^{x}$ and $V=-J_{z} \sum_{\boldsymbol{r}} \sigma_{\boldsymbol{r}}^{z} \sigma_{\boldsymbol{r}+\boldsymbol{e}_{z}}^{z}$.

Since each term of $V$ flips a pair of spins on neighboring chains, it is necessary to apply the perturbation at least $L$ times on an $L \times L$ lattice to flip all spins of a pair of neighboring chains and reach another ground state. So the gap is expected to scale as $\Delta / J_{x}=a\left(b J_{z} / J_{x}\right)^{L}$. One can be more precise though and determine the constants $a$ and $b$ from high order perturbation theory. To first significant order in $J_{z} / J_{x}$, the gap $\Delta$ is given by the gap between lower and higher eigenvalues of the effective Hamiltonian

$$
H_{\mathrm{eff}}^{(L)}=\sum_{\nu_{1}>0} \cdots \sum_{\nu_{L-1}>0} \frac{P_{0} V P_{\nu_{1}} V \cdots V P_{\nu_{L-1}} V P_{0}}{\left(E_{0}-E_{\nu_{1}}\right) \cdots\left(E_{0}-E_{\nu_{L-1}}\right)},
$$

where $P_{\nu}$ is the projector on the $\nu$-th eigenspace of $H_{0}$. This eigenspace has an energy $E_{\nu}=-J_{x} N+4 \nu J_{x}$, where 


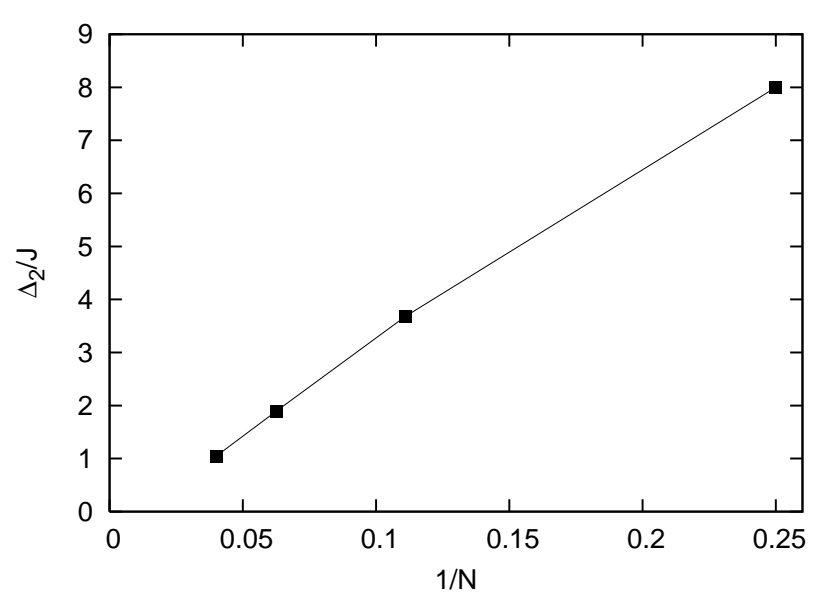

FIG. 3: Gap $\Delta_{2}$ (see text) as a function of the number of sites $N$.

$\nu$ can take the values $\nu=0,1, \cdots, L / 2$ when $L$ is even and $\nu=0,1, \cdots,(L-1) / 2$ when $L$ is odd. Let $\{|\nu, k\rangle$ : $k=0,1, \cdots\}$ be a basis of the $\nu$-th eigenspace of $H_{0}$. We have to evaluate

$$
\begin{aligned}
\langle 0, l| V & P_{\nu_{1}} V \cdots V P_{\nu_{L-1}} V|0, k\rangle \\
= & \left(-J_{z}\right)^{L} \sum_{i_{1}, j_{1}} \cdots \sum_{i_{L}, j_{L}}\langle 0, l| \sigma_{i_{1}, j_{1}}^{z} \sigma_{i_{1}, j_{1}+1}^{z} P_{\nu_{1}} \\
& \cdots P_{\nu_{L-1}} \sigma_{i_{L}, j_{L}}^{z} \sigma_{i_{L}, j_{L}+1}^{z}|0, k\rangle .
\end{aligned}
$$

For $L>2$, the only contribution to $\Delta$ arise when the $\sigma$ product flips two neighboring lines with $r_{z}=j$ and $r_{z}=j+1$. It corresponds to $j_{1}=\cdots=j_{L}=j$ and $i_{1}=p(1), \cdots, i_{L}=p(L)$ with $p \in S_{L}$ and $S_{L}$ the set of $L$ ! permutations. The ground state $|0, k\rangle$ contains only ferromagnetic lines in the $x$ or $-x$ direction. So, for $m<$ $L$, the state $\sigma_{p(m+1), j}^{z} \sigma_{p(m+1), j+1}^{z} \cdots \sigma_{p(L), j}^{z} \sigma_{p(L), j+1}^{z}|0, k\rangle$ cannot be in the ground state manifold of $H_{0}$, but it must be in one of the excited eigenspaces, say the $\nu_{m}(p)$-th. Then we can write:

$$
\begin{aligned}
\left\langle 0, l\left|V P_{\nu_{1}} V \cdots V P_{\nu_{L-1}} V\right| 0, k\right\rangle & \\
= & \left(-J_{z}\right)^{L} \sum_{p \in S_{n}} \sum_{j}\langle 0, l| \sigma_{p(1), j}^{z} \sigma_{p(1), j+1}^{z} \\
& \cdots \sigma_{p(L), j}^{z} \sigma_{p(L), j+1}^{z}|0, k\rangle \prod_{m=1}^{L-1} \delta_{\nu_{m}, \nu_{m}(p)} .
\end{aligned}
$$

Using $E_{\nu}=E_{0}+4 \nu J_{x}$ and rearranging the $\sigma^{z}$ 's, we get:

$$
\begin{aligned}
\left\langle 0, l\left|H_{\mathrm{eff}}^{(L)}\right| 0, k\right\rangle & \\
= & -\frac{J_{z}^{L}}{\left(4 J_{x}\right)^{L-1}} \sum_{p \in S_{n}} \frac{1}{\nu_{1}(p) \cdots \nu_{L-1}(p)} \\
& \left\langle 0, l\left|\sum_{j} \sigma_{1, j}^{z} \sigma_{1, j+1}^{z} \cdots \sigma_{L, j}^{z} \sigma_{L, j+1}^{z}\right| 0, k\right\rangle .
\end{aligned}
$$

Next, we note that, in terms of the pseudo-spin $\tau_{j}^{z}=$ $\sigma_{1, j}^{z} \cdots \sigma_{L, j}^{z}$, the operator $\sum_{j} \sigma_{1, j}^{z} \sigma_{1, j+1}^{z} \cdots \sigma_{L, j}^{z} \sigma_{L, j+1}^{z}$ is nothing but the Hamiltonian of the one-dimensional Ising model. So, the gap between the lowest and highest eigenvalues is $\lambda_{\max }-\lambda_{\min }=2 L$ when $L$ is even and $\lambda_{\max }-\lambda_{\min }=2(L-1)$ when $L$ is odd. Finally, if we define $P(L)$ by

$$
P(L)=\sum_{p \in S_{n}} \frac{1}{\nu_{1}(p) \cdots \nu_{L-1}(p)}
$$

the gap $\Delta$ becomes

$$
\Delta / J_{x}= \begin{cases}8 L P(L)\left(J_{z} / 4 J_{x}\right)^{L} & \text { if } L \text { is even } \\ 8(L-1) P(L)\left(J_{z} / 4 J_{x}\right)^{L} & \text { if } L \text { is odd. }\end{cases}
$$

The dominant behaviour of $P(L)$ has been determined numerically (see Fig. 4). It turns out that $L P(L) \simeq$ $\exp (0.754 L-0.694)$, which leads to:

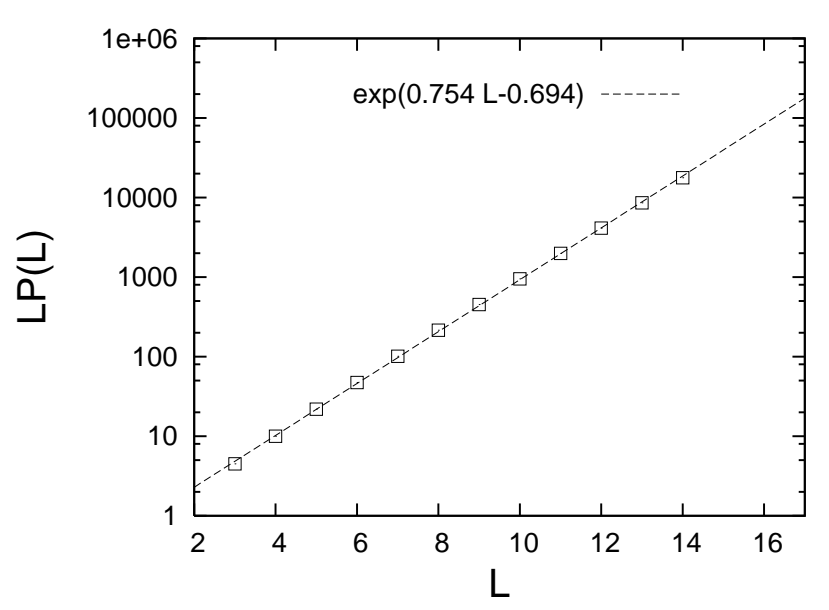

FIG. 4: log-linear plot of $L P(L)$ versus the linear lattice size $L$. The squares are the results obtained numerically, and the dotted line is an exponential fit.

$$
\Delta / J_{x}= \begin{cases}3.997\left(0.531 J_{z} / J_{x}\right)^{L} & \text { if } L \text { even } \\ 3.997(1-1 / L)\left(0.531 J_{z} / J_{x}\right)^{L} & \text { if } L \text { odd }\end{cases}
$$

It has the correct $\left(J_{z} / J_{x}\right)^{L}$ behavior which corresponds to the exact result for the two-line system [7]. So, when $\left(J_{z} / J_{x}\right) \ll 1$, this approximation predicts $\Delta \rightarrow 0$ in the thermodynamic limit. Moreover, since $0.531\left(J_{z} / J_{x}\right)<1$ even for $J_{z} / J_{x}=1$, it seems likely that this scaling will remain true up to the symmetric limit $J_{x}=J_{z}$. As we shall see, this is confirmed by the Green's function Monte Carlo results.

\section{Green's function Monte Carlo}

If the Hamiltonian of a model has only non-povitive off-diagonal matrix elements, which is the case here, the 
Green's function Monte Carlo method [12] allows one to calculate the ground-state energy of a given symmetry sector by using a stochastic approach. The algorithm we have used is the implementation with a fixed number of walkers described in detail by Calandra and Sorella [12], and the guiding function is given by:

$$
\left|\psi_{G}\right\rangle=\exp \left(\frac{1}{2} \sum_{\boldsymbol{r}, \boldsymbol{r}^{\prime}} v_{\boldsymbol{r}, \boldsymbol{r}^{\prime}} \sigma_{\boldsymbol{r}}^{z} \sigma_{\boldsymbol{r}^{\prime}}^{z}\right)\left|F_{x}\right\rangle,
$$

where $\left|F_{x}\right\rangle$ is the ferromagnetic state in $x$ direction such that $\sigma_{\boldsymbol{r}}^{x}\left|F_{x}\right\rangle=\left|F_{x}\right\rangle \forall \boldsymbol{r}$. The parameters $v_{\boldsymbol{r}, \boldsymbol{r}^{\prime}}$ were determined in order to minimize the energy of the guiding function. The ground state energy has been determined in each sector $\left(p_{1}, \cdots, p_{L}\right)$ separately, which corresponds to the energy of the first $2^{L}$ states of the full Hilbert space (the states coming from the limit $J_{z}=0$ ground state in Fig. (2), and gives access to the gap $\Delta(\theta)$.

Let us first discuss the scaling of the energy per site as a function of the system size. As can be seen in Fig. energy per site is strongly size dependent up to a certain size $\left(8 \times 8\right.$ for $\left.J_{x}=J_{z}\right)$, and is very little size dependent for larger clusters. This indicates that strong finite-size effects are to be expected, especially close to the symmetric point, justifying the use of quantum Monte Carlo to get information on large clusters.

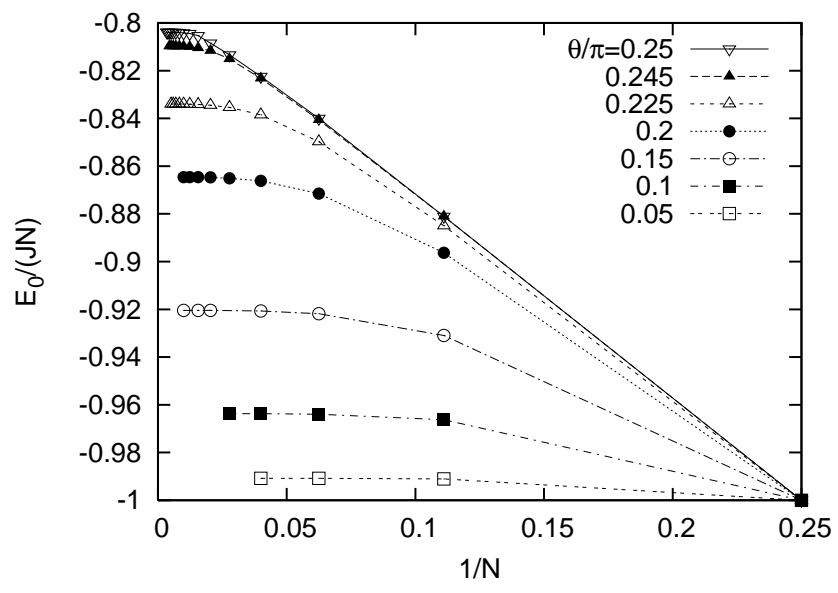

FIG. 5: Ground-state energy $E_{0}$ versus the number of sites $N$ for various values of the asymmetry parameter $\theta$.

Fig. 6 shows a log-linear plot of the gap $\Delta$ versus the linear lattice size $L$ for various values of the asymmetry parameter $\theta$. Perturbation theory predicts that the scaling of $\Delta$ is given by a power law $\Delta / J_{x} \propto \alpha^{L}$, with $\alpha \simeq 0.531 J_{z} / J_{x}$. To check this prediction, we have fitted the results of Fig. [6 with a straight line for each value of $\theta$, keeping only sizes beyond which the scaling is approximately linear. The values of $\alpha$ deduced from this fit are plotted in Fig. [ 7 as a function of $J_{z} / J_{x}$. Remarkably, the relation between $\alpha$ and $J_{z} / J_{x}$ is quite linear up to $J_{x}=J_{z}$, which seems to justify the perturbation theory in this limit. Moreover, a fit gives $\alpha \simeq 0.55 J_{z} / J_{x}$,

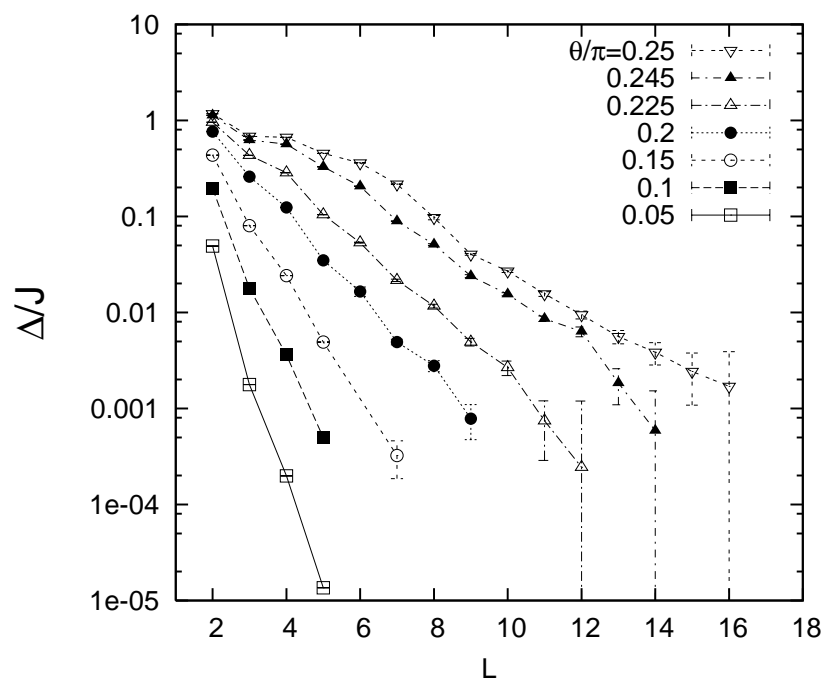

FIG. 6: Gap $\Delta$ versus linear lattice size $L$ for various values of the asymmetry parameter $\theta$. For $L=2,3,4,5$ the results were obtained from exact diagonalization.

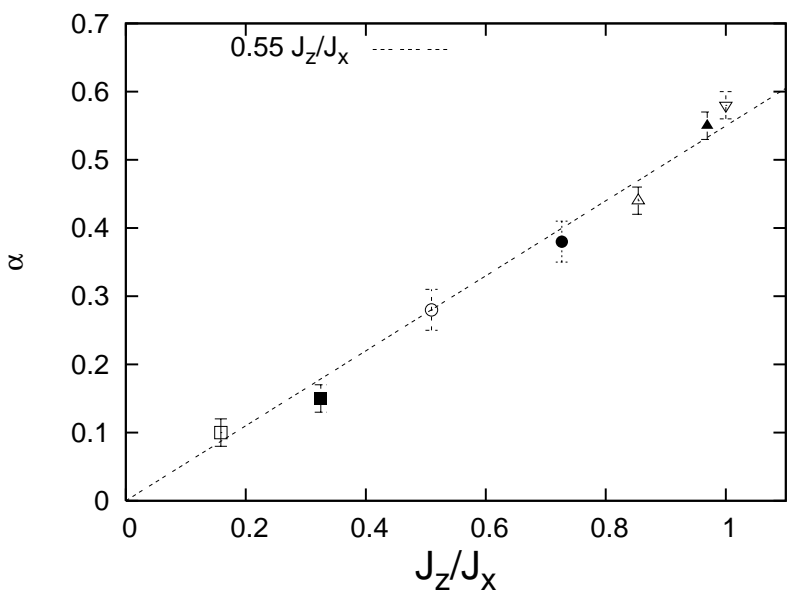

FIG. 7: $\alpha$ versus $J_{z} / J_{x}$, where $\alpha$ is the gap scaling constant $\Delta \sim \alpha^{L}$. The symbols are the results obtained with Green's function Monte Carlo and the dotted line is fitted to these values.

in good agreement with the perturbation theory prediction $\alpha \simeq 0.531 J_{z} / J_{x}$. In fact, for small $J_{z} / J_{x}$, even the prefactor and the even-odd effect predicted by the perturbation theory agree with the results of Fig. 6. All these results lead us to the conclusion that the gap $\Delta$ indeed follows a power law $\Delta / J \sim \alpha^{L}$, with $\alpha<1$ when $J_{z} \leq J_{x}$, implying that $\Delta \rightarrow 0$ in the thermodynamic limit. Thus the $2^{L}$ states coming from the limit $\theta=0$ $\left(J_{z}=0\right)$ ground state collapse in the thermodynamic limit, as long as $J_{z} \leq J_{x}$. Using the rotation symmetry $R_{y}\left(\frac{\pi}{2}\right)$, the same result holds true for the $2^{L}$ states coming from the limit $\theta=\frac{\pi}{2}$ (i.e., $J_{x}=0$ ) ground state, as long as $J_{z} \geq J_{x}$. The conclusion is that there are $2^{L}$ states collapsing exponentially fast onto each other when 
$J_{x} \neq J_{z}$, and at least a $2 \times 2^{L}-2$ when (i.e., $J_{x}=J_{z}$ ). As we argued above, the actual number is very probably actually equal to $2 \times 2^{L}$, in agreement with the semiclassical analysis.

\section{GENERAL SPIN}

The symmetry arguments used for spin- $1 / 2$ can be easily extended to arbitrary spins. Let us consider the system with $N$ spins $S$ on a $L \times L$ lattice described by the compass model Hamiltonian

$$
H=-\sum_{\boldsymbol{r}}\left(J_{x} S_{\boldsymbol{r}}^{x} S_{\boldsymbol{r}+\boldsymbol{e}_{x}}^{x}+J_{z} S_{\boldsymbol{r}}^{z} S_{\boldsymbol{r}+\boldsymbol{e}_{z}}^{z}\right) .
$$

It is straightforward to check that the generalizations of the $P_{j}$ and $Q_{i}$ defined by

$$
\begin{aligned}
& P_{j}=\prod_{l} i e^{-i \pi S_{l, j}^{z}} \\
& Q_{l}=\prod_{j} i e^{-i \pi S_{l, j}^{x}}
\end{aligned}
$$

for integer spins and by

$$
\begin{aligned}
P_{j} & =\prod_{l} e^{-i \pi S_{l, j}^{z}} \\
Q_{l} & =\prod_{j} e^{-i \pi S_{l, j}^{x}}
\end{aligned}
$$

for half-integer spins commute with the Hamiltonian. However, it is easy to check that the commutator $\left[Q_{i}, P_{j}\right]$ vanishes for integer spins, whereas it does not vanish for half-integer spins $\left(\left\{Q_{i}, P_{j}\right\}=0\right)$. Indeed, the only terms in the product which do not trivialy commute are those on the site $(i, j)$. So we just have to show that $\left[e^{-i \pi S_{i, j}^{x}}, e^{-i \pi S_{i, j}^{z}}\right]=0$ for integer spins and $\left\{e^{-i \pi S_{i, j}^{x}}, e^{-i \pi S_{i, j}^{z}}\right\}=0$ for half-integer spins. The operator $e^{-i \pi S_{i, j}^{z}}=R_{z}(\pi)$ corresponds to the spin rotation by an angle $\pi$ about the $z$ axis acting at site $(i, j)$. Applying this rotation to $e^{-i \pi S_{i, j}^{x}}$ gives

$$
\begin{aligned}
R_{z}^{-1}(\pi) e^{-i \pi S_{i, j}^{x}} R_{z}(\pi) & =\sum_{n} \frac{(-i \pi)^{n}}{n !} R_{z}^{-1}(\pi)\left(S_{i, j}^{x}\right)^{n} R_{z}(\pi) \\
& =\sum_{n} \frac{(-i \pi)^{n}}{n !}\left(-S_{i, j}^{z}\right)^{n} \\
& =e^{i \pi S_{i, j}^{z}}
\end{aligned}
$$

But for integer spins, $e^{i \pi S_{i, j}^{z}}=e^{-i \pi S_{i, j}^{z}}$, while for halfinteger spins, $e^{i \pi S_{i, j}^{z}}=-e^{-i \pi S_{i, j}^{z}}$, which terminates the proof. This has two important consequences. First of all, in Douçot et al.'s argument, the fact that $\left[Q_{i}, P_{j}\right] \neq 0$ was crucial to show that each state was two-fold degenerate. We thus expect that for all integer spins, the eigenstates are generically non-degenerate. Besides, for integer spins, since all $Q_{i}$ 's commute with all $P_{j}$ 's, one can use all these symmetries simultaneously, leading to $2^{2 L}$ different symmetry sectors.

We have checked these predictions for $S=1$ with exact diagonalizations of clusters of size $L=2$ and $L=3$ (see Fig. 8). Indeed, the ground state is non-degenerate
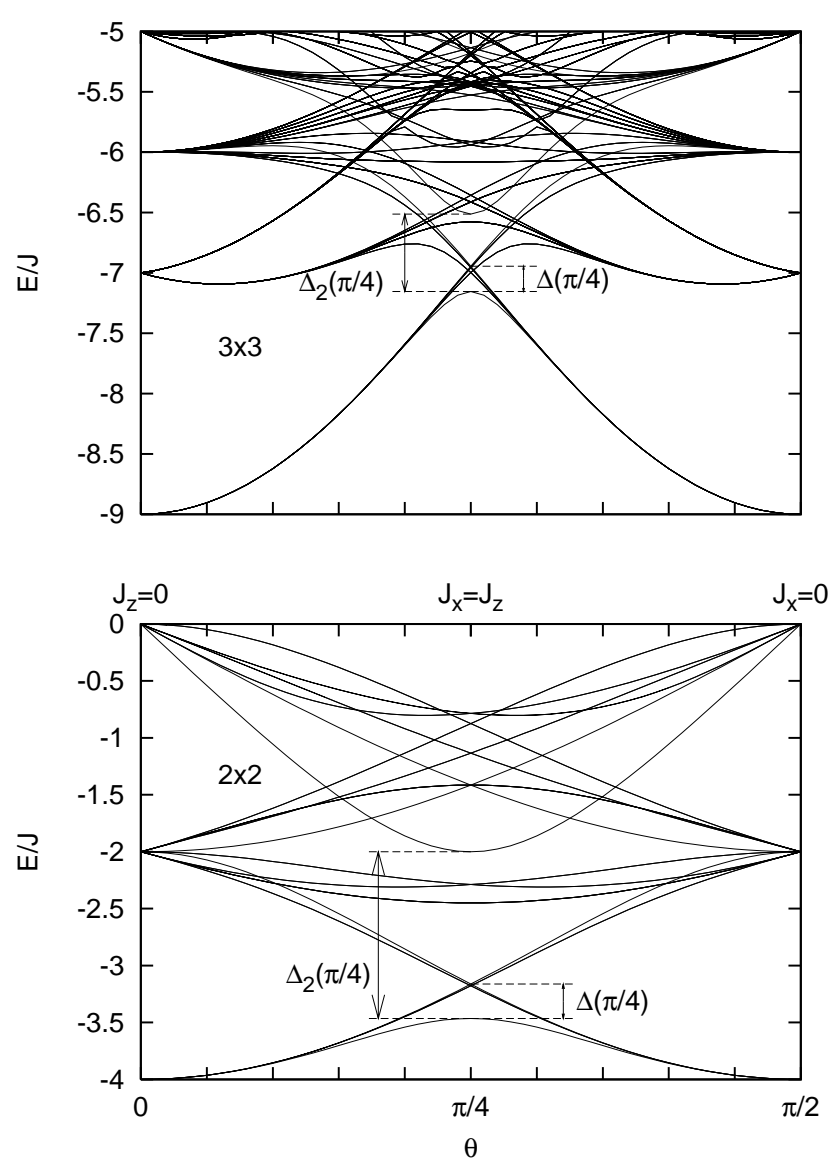

FIG. 8: Spectrum versus asymmetry parameter $\theta$ for linear lattice sizes $L=2$ and $L=3$ obtained by exact diagonalization. $J_{x}=J \cos \theta$ and $J_{z}=J \sin \theta$.

for all $J_{x}, J_{z} \neq 0$, and it is in the only symmetry sector $p_{1}=\cdots=p_{L}=q_{1}=\cdots=q_{L}=(-1)^{L}$ connecting the ground states in the limits $J_{x}=0$ and $J_{z}=0$. The $J_{z}=0$ (resp. $J_{x}=0$ ) ground state $2^{L}$-fold degeneracy is partially lifted by the $J_{z}$ (resp. $J_{x}$ ) term of the Hamiltonian, creating a gap $\Delta$. As in the spin- $1 / 2$ model, the gap between the ground state and the first excited state which has the same degeneracy and is in the same symmetry sector as the ground state is denoted by $\Delta_{2}$. The gaps $\Delta$ and $\Delta_{2}$ are smaller for $L=3$ than for $L=2$, and one can conjecture that these gaps go to zero in the thermodynamic limit. In this case, the ground state would have the same degeneracy as for spin-1/2. A definite conclusion would clearly require to study larger clusters though. 


\section{CONCLUSION}

Using a variety of approaches, we have obtained a coherent picture of the zero-temperature properties of the quantum compass model. On a finite cluster, we have confirmed that all eigenstates of the spin-1/2 model are at least two-fold degenerate, a result that we have extended to arbitrary half-integer spins, while they are not necessarily degenerate for integer spins. However, the degeneracy that remains when thermal or semi-classical quantum fluctuations are introduced, namely the possibility to flip the spins along lines or columns, is still present as a manifold of states which collapse exponentially fast onto the ground state upon increasing the size of the lattice. This was already known to be the case for the asymmetric case not too close to $J_{x}=J_{z}$. Thanks to extensive quantum Monte Carlo simulations, we have shown that this remains true up to the symmetric case $J_{x}=J_{z}$, and that the the number of these states $\left(2^{L}\right.$ when $J_{x} \neq J_{z}, 2 \times 2^{L}$ when $J_{x}=J_{z}$ ) agrees with the degeneracy predicted by the semi-classical analysis.

Physically, this has two consequences. Regarding orbital fluctuations in Mott insulators, in which case the symmetric version of the model seems more appropriate, the present results confirm the absence of true orbital long-range order for quantum spins in the thermodynamic limit even at zero temperature. Nevertheless, as in the case of thermal fluctuations for classical spins, the possibility to choose between the $x$ and $z$ directions should still lead to a finite-temperature Ising transition.

Regarding Josephson junction arrays, one of the important issues is to ensure that the two-fold degenerate ground state is well protected by a gap to all excited states. As noticed by Douçot et al., this requires to work with not too large systems if a family of states collapse onto the ground state in the thermodynamic limit, as they already showed to be the case for $J_{x} \ll J_{z}$. Their results suggested however that there might be a quantum phase transition to a gapped phase around $J_{x}=J_{z}$ in which the gap to all excited states would remain finite even in the thermodynamic limit. This possibility is clearly ruled out by the present results.

\section{Acknowledgments}

We thank Mike Ma for enlightning discussions about the symmetries of the model, Arnaud Ralko for useful discussions about Green's function Monte Carlo, and Benoît Douçot for a critical reading of the manuscript. This work was supported by the Swiss National Fund and by MaNEP. F.B. was supported by INFM and by MIUR (COFIN 2004).
[1] K. I. Kugel and D. I. Khomskii, Sov. Phys. Usp. 25, 231 (1982).

[2] K. I. Kugel and D. I. Khomskii, Sov. Phys. JETP 37, 725 (1973).

[3] M. Mostovoy and D. I. Khomskii, Phys. Rev. Lett. 89, 227203 (2002).

[4] F. Mila, Phys. Rev. Lett. 81, 2356 (1998).

[5] K. Hida, J. Phys. Soc. Jpn 70, 3673 (2001); D. C. Cabra, M. D. Grynberg, P. C. W. Holdsworth, and P. Pujol, Phys. Rev. B 65, 094418 (2002); J. Schulenburg, A. Honecker, J. Schnack, J. Richter, and H.-J. Schmidt, Phys. Rev. Lett. 88, 167207 (2002); A. Honecker, J. Schulenburg, and J. Richter, J. Phys.: Condens. Matter 16, S749 (2004).

[6] D. C. Cabra, private communication.

[7] B. Douçot, M. V. Feigel'man, L. B. Ioffe, and A. S. Iose- levitch, Phys. Rev. B 71, 024505 (2005).

[8] Z. Nussinov, M. Biskup, L. Chayes, and J. van den Brink, Europhys. Lett. 67, 990 (2004).

[9] A. Mishra, M. Ma, F-C. Zhang, S. Guertler, L-H. Tang, and S. Wan, Phys. Rev. Lett. 93, 207201 (2004).

[10] Z. Nussinov and E. Fradkin, cond-mat/0410720

[11] P. Chandra and B. Douçot, Phys. Rev. B 38, 9335 (1988).

[12] Nandini Trivedi and D. M. Ceperley, Phys. Rev. B 41, 4552 (1990). In particular, we used the algorithm described by M. Calandra and S. Sorella, Phys. Rev. B 57, 11446 (1998).

[13] Unfortunately, this state is not accessible to the quantum Monte Carlo algorithm, which only gives access to the ground-state energy of a given sector. 